\title{
El Mundo de una mujer llamada Elba hace 9300 años
}

\author{
The world of a woman named Elba 9300 years ago
}

\author{
VIDAL ROMANÍ J.R., GRANDAL D’ANGLADE, A. y VAQUEIRO RODRÍGUEZ, M. ${ }^{1}$ \\ (1) Instituto Universitario de Geología. Universidad de Coruña. Campus de Elviña, Edificio ESCI. \\ Coruña, Galicia, España.
}

https://doi.org/10.0.70.59/cadlaxe.2017.39.0.7361

\begin{abstract}
Resumen.
Elba, la que viene de las montañas, ha sido el nombre celta elegido para los restos de una joven a la que sorprendió la muerte viajando con 3 uros hace 9300 años en un camino de montaña entre Courel y Ancares (Galicia, España). El volumen 39 de CLXL presenta los datos morfológicos y cronológicos de la cueva donde se encontró a Elba. También se publica el estudio genético, isotópico y forénsico de sus restos y una reproducción de su cara y cabeza, ambas obtenidas utilizando las técnicas de restauración habituales en la ciencia forense.
\end{abstract}

\begin{abstract}
Elba, the woman that comes from the mountains is the english translation of the Celtic name that has been chosen for the remains found on an old path that goes from Courel to Ancares (Galice, Spain) and that correspond to a young woman who surprised the death traveling with 3 uros 9300 years ago. It presents the description of the cave where the remains were found, its genetic and isotopic study, a forensic study of its remains as well as a reproduction of its face obtained by the usual restoration techniques.
\end{abstract}




\section{COUREL EN EL TIEMPO DE ELBA}

Se aportan datos para reconstruir un hecho singular acaecido hace 9300 años durante un viaje a través de las Serras de O Courel y Ancares en Galicia. En contra de lo habitual en estos casos la tragedia quedó registrada hasta el día de hoy en un tipo de archivo muy específico que solo el nivel de conocimiento del que disponemos en el siglo XXI ha permitido ahora interpretar. La protagonista de nuestra historia era una joven gallega como lo indican los datos genéticos (González Fortes et al. 2017) y forénsicos (Serrulla Sanin 2017) que convenientemente estudiados permiten asegurar que la procedencia de Elba era cercana (González Fortes et al. 2017) al lugar donde se hallaron sus restos en Cova do Uro (Chan do Lindeiro). Genéticamente Elba no se relaciona con ninguna migración humana desde lugares remotos, muy al contrario Elba era "gente de aquí". Incluso la reconstrucción del aspecto de la protagonista de esta historia permite afirmar que de poder verla ahora mismo tal como era se nos pasaría desapercibida (Sanin y Serrulla 2017), tal es la similaridad de su aspecto físico con las actuales mujeres de los pueblos de montaña en la Galicia interior. Por lo demás del análisis forense (Serrulla y Sanin 2017; Sanin y Serrulla (2017) de los restos de Elba, aun correspondiendo solo a parte del esqueleto, se han obtenido datos como estatura, tamaño y forma de su cabeza, así como numerosos signos de estrés nutricional, huellas de traumatismos cráneo encefálicos previos a su muerte que, todos ellos, hablan elocuentemente la dureza de la vida en esas zonas interiores de montaña desde hace 10 milenios y que se han mantenido prácticamente constantes hasta épocas muy recientes. También nos dicen que la mujer gallega está desde siempre ocupando la primera línea de la lucha por la supervivencia, aun en ambientes extremos como debió ser Galicia en los tiempos de Elba. La combinación de los datos forenses obtenidos a partir de su esqueleto con los genéticos (González Fortes et. al, 2017), ha permitido además añadir a los deducidos de los huesos otros como color del pelo, piel y ojos. Y a partir de aquí utilizando un método clásico de reconstrucción (Sanin y Serrulla (2017) se ha obtenido una réplica, a tamaño natural, de la cabeza de Elba.

Lo que hemos llegado a conocer a partir del estudio de sus restos es que se trataba de una mujer joven que cuando murió, tenía entre los 20 y los 40 años y que se desplazaba, como debía hacerlo habitualmente, siguiendo un sendero, que aun hoy existe, entre la actuales Serra do Courel y Serra de Ancares en compañía de tres uros: uno de mayor edad y tamaño, siendo los otros dos indivíduos mas jóvenes y de menor talla. Los restos de la mujer y los 3 uros se encontraron todos juntos en el interior de una dolina perteneciente al sistema cárstico de la Cova do Uro. El origen de esta cavidad (VaqueiroRodríguez et al., 2017) como el de la mayor parte de las cuevas de O Courel es mixto al haberse formado por neotectónica (principalmente) y por disolución (secundariamente) aunque la edad exacta para la formación de la cueva no es conocida (Cuaternario s.1.). Sin embargo sin duda que Cova do Uro es mucho mas antigua que los restos óseos fósiles encontrados en ella. Otra cuestión es la de la edad de formación de la dolina donde se encontraron los restos de Elba y los 3 uros. La apertura de la dolina al exterior debió suceder posiblemente hace 9300 años que es como podríamos interpretar el hecho de que Elba y los uros aparecieran en 
el fondo de la cavidad, es decir: no existía un relleno de la dolina antes de la caída de Elba y los uros. También se explicaría así la coincidencia en la edad de apertura de la dolina y los restos fósiles el hecho de que el trazado del camino coincidiera con el hueco de la dolina pues de lo contrario el camino se hubiera desviado antes impidiendo la caída de Elba y los uros. Por su parte la cronología, realizada con radiocarbono, de los restos ha confirmado una misma edad para todos, uros y Elba lo que sugiere que viajaban juntos (Grandal dAnglade y Vidal-Gorosquieta 2017) ya que la relación isotópica del $\mathrm{Sr} 86 /$ Sr87 detectado en Elba y los 3 uros indica un lugar de procedencia ajeno al lugar donde se encontraron los restos de todos ellos. Es por ello que basándose en esta razón, se interpreta esta asociación en el tiempo y en el espacio como una prueba de un caso incipiente de pastoralismo pues es menos creíble que la doble coincidencia sea fruto del azar. La situación de la dolina a poco mas de un metro de distancia de la traza del camino actual nos inclina a pensar que muchas veces los senderos que aun funcionan en las montañas gallegas existen desde hace mas tiempo del que imaginamos. Éste camino en concreto aunque tiene casi 10.000 años de antigüedad con las modificaciones actuales, continúa funcionando. Y esta afirmación la fundamentamos en la caída de 4 seres vivos en una dolina situada muy próxima al trazado del camino actual (Vaqueiro et al. 2017), que funcionó como trampa sedimentaria para Elba y los uros y por ello lo más lógico es que los protagonistas de nuestra historia pasaran habitualmente por la zona. Descartamos que, por ejemplo, estuvieran pastando en la zona porque se trata de un monte rocoso, con escasa vegetación y porque las zonas de pastos actuales se sitúan a cotas mas bajas que la de la boca de la dolina. Por otra parte el estudio de la procedencia de los uros (Vidal-Gorosquieta et al. 2016), indica que aunque son de la zona, proceden de entornos geológicos muy distintos,. Este dato refuerza una vez mas la hipótesis del tránsito y del pastoralismo por lo que la hipótesis de una muerte accidental por caída en la dolina parece la mas lógica. En algunos casos en los mapas de países históricamente antiguos se conserva aun la conexión histórica entre el indicativo escrito en el mapa y las viejas rutas seguidas por los antecesores de la población actual. Es el caso de los caminos guanches en Canarias y el camino del Inca en Perú. El caso de Elba llega mucho mas allá de la memoria escrita o aun de la conservada en las leyendas transmitidas, a veces solo oralmente.

Otro hecho que se puede deducir del hallazgo es que la, aparentemente, caída simultánea en la dolina de los 3 uros y la joven Elba indica un viaje al final del invierno o tal vez al inicio de una primavera fría con el paisaje recubierto quizás por la última nevada del año. Un episodio meteorológico breve, aunque lo suficientemente frío, como para producir una nevada, no demasiado copiosa que la joven Elba con su pequeña estatura (entre 150 y $152 \mathrm{~cm}$ ) no renunciara a cruzar la Sierra llevando los 3 uros. El viaje se hizo siguiendo un sendero cuyo trazado discurre por la parte mas alta del valle, lo que garantizaría también condiciones de mayor seguridad: mayor visibilidad y control del entorno y de los posibles enemigos y un desplazamiento mas fácil en cualquier época del año, aunque especialmente durante el invierno pues el espesor de la nieve en la parte superior del valle nunca sería tan grande como en el fondo del valle. A pesar de todo durante aquella primavera de hace 
10 milenios la nevada fue lo suficiente escasa como para no desanimar a Elba a cruzar la Sierra aunque si lo suficientemente persistente como para disimular la boca de la dolina que además estaría cubierta por la vegetación. Todo se confabuló para que Elba y los uros cayeran a la dolina para no volver a salir hasta después de transcurridos casi 10 milenios.

En el resto de O Courel las cosas eran un poco diferentes a como las vemos en la actualidad. La laguna de A Lucenza, (Fig. 1), una antigua cubeta de sobreexcavación glacial que llegó a alcanzar mas de 10

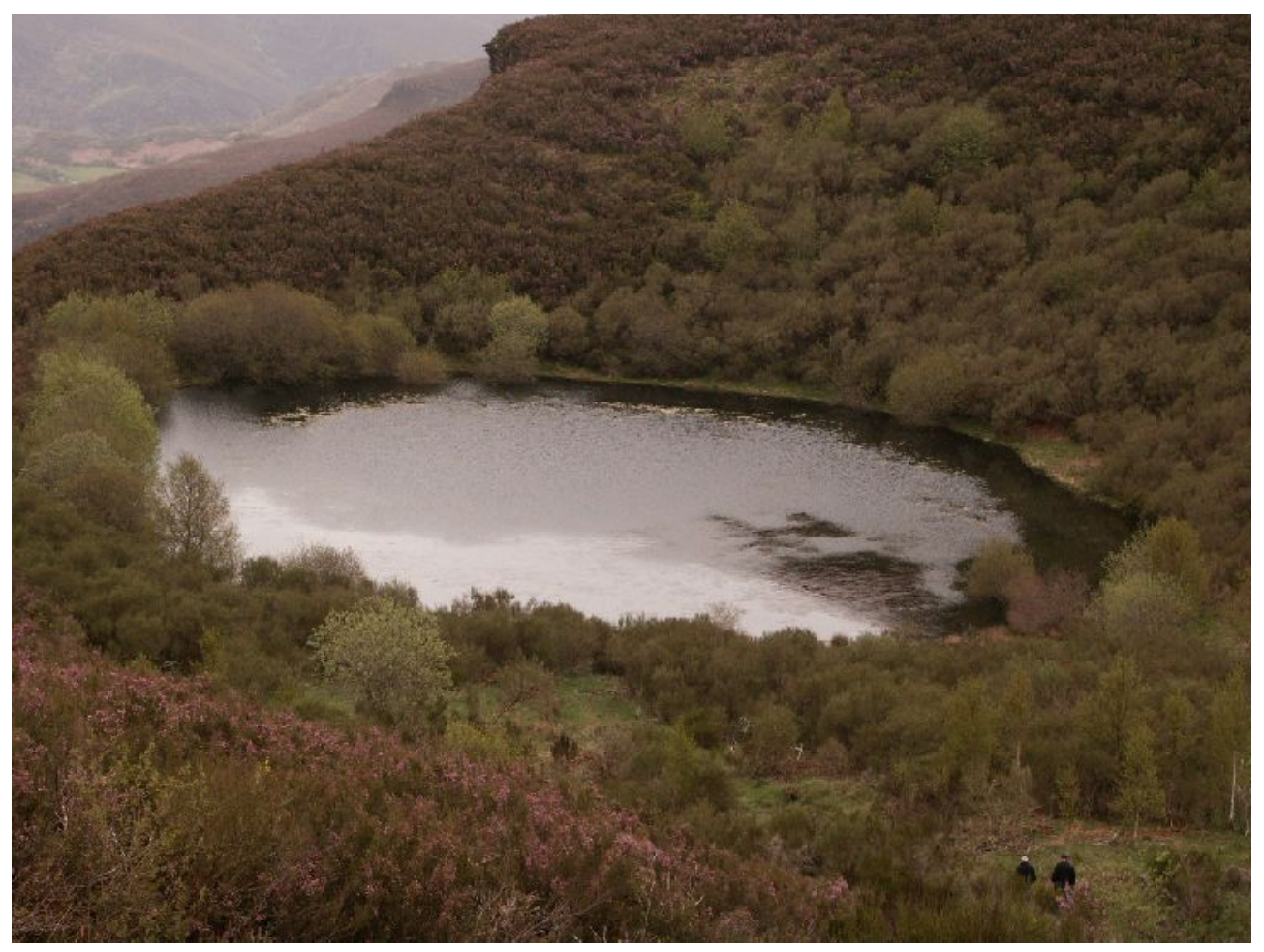

Fig. 1. La laguna de A Lucenza

metros de profundidad durante las fases mas frías de la última etapa glacial, estaba ya empezando a rellenarse de sedimentos (el agua habría pasado de 10 al final de la última fase glacial a 4.5 metros de profundidad) (Santos et al. 2000; Railsback et al., 2011). Y ha sido precisamente gracias a ese registro sedimentario como se ha podido reconstruir el tipo de vegetación que existía en la zona por donde se movían Elba y sus uros hace 10 milenios: un área con un desarrollo arbóreo evidente donde los abedules mostraban un breve máximo y el roble comenzaba a expandirse. También estaban presentes avellanos, alisos, olmos y pequeños bosquetes de pinos así como un mon- 
te bajo formado por herbáceas, artemisia, ericáceas, rumex, etc., que también comenzaban a disminuir pues desaparecen virtualmente de la zona en el último tercio del Holoceno (Santos et al. 2000). Por esta razón el paisaje vegetal sería bastante parecido al actual con la excepción de las especies de reciente introducción. Quizás lo mas notable de lo aportado por los datos paleobotánicos es la identificación en los suelos de aquella etapa de una gran abundancia de partículas de carbón confirmando la existencia de fuegos forestales (Santos et al. 2000). Este dato hasta hace poco interpretado como causa- do por incendios debidos a fenómenos naturales (tormentas eléctricas) al no haberse detectado polen de cereales, a la luz de los nuevos datos aportados por la presencia de Elba y sus uros puede ser ahora entendido como debido, al igual que sucede en el momento actual, a la acción de los pastores que buscaban así mediante esos fuegos tempranos en el inicio de la primavera del rebrote de la vegetación. Mas de un milenio después (Steelman et al., 2017) los ocupantes de Cova Eirós realizarían las insólitas pinturas descritas en ese punto tan cercano a la dolina de la Cova do Uro.

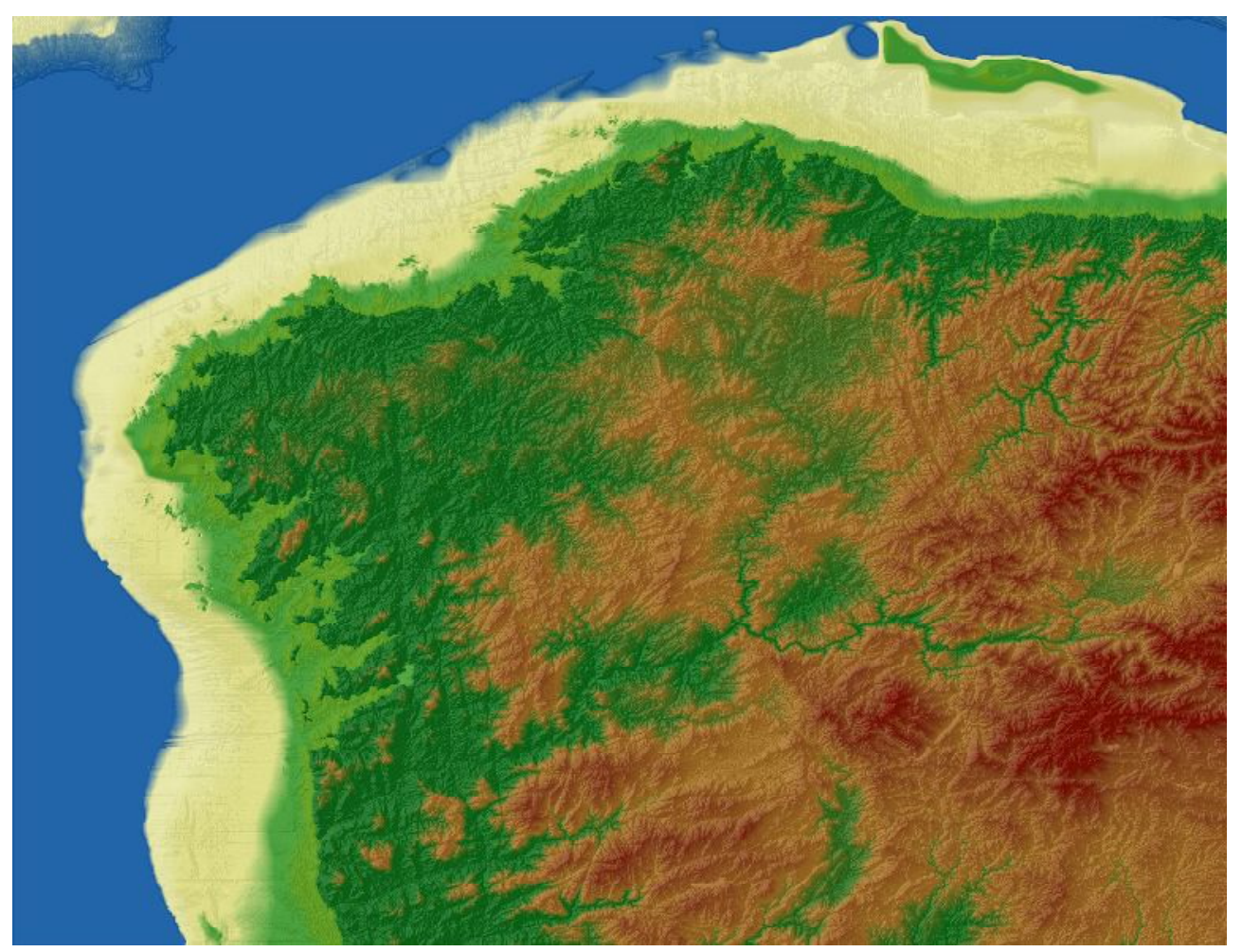

Fig. 2. Paleogeografía de la Galicia de Elba 


\section{EL RESTO DE GALICIA EN EL TIEMPO DE ELBA}

Por lo que se refiere al resto de Galicia especialmente en la zona costera (Figura 2) que es donde las cosas eran muy diferentes a la actualidad, el nivel del mar se situaba unos 32 metros por debajo del actual indicando que la última deglaciación había comenzado ya (hacía 5000 años) aunque las rías aun eran valles fluviales (Vidal Romaní 2015) pues el mar estaba estacionado en la boca de las rías como prueban los frecuentes hallazgos de restos de bosques fósiles con la misma edad que Elba en zonas como Cíes, Patos (Pontevedra), Seselle, Ponzos (Coruña) y Arealonga (Lugo) (Figs. 3 y 4).

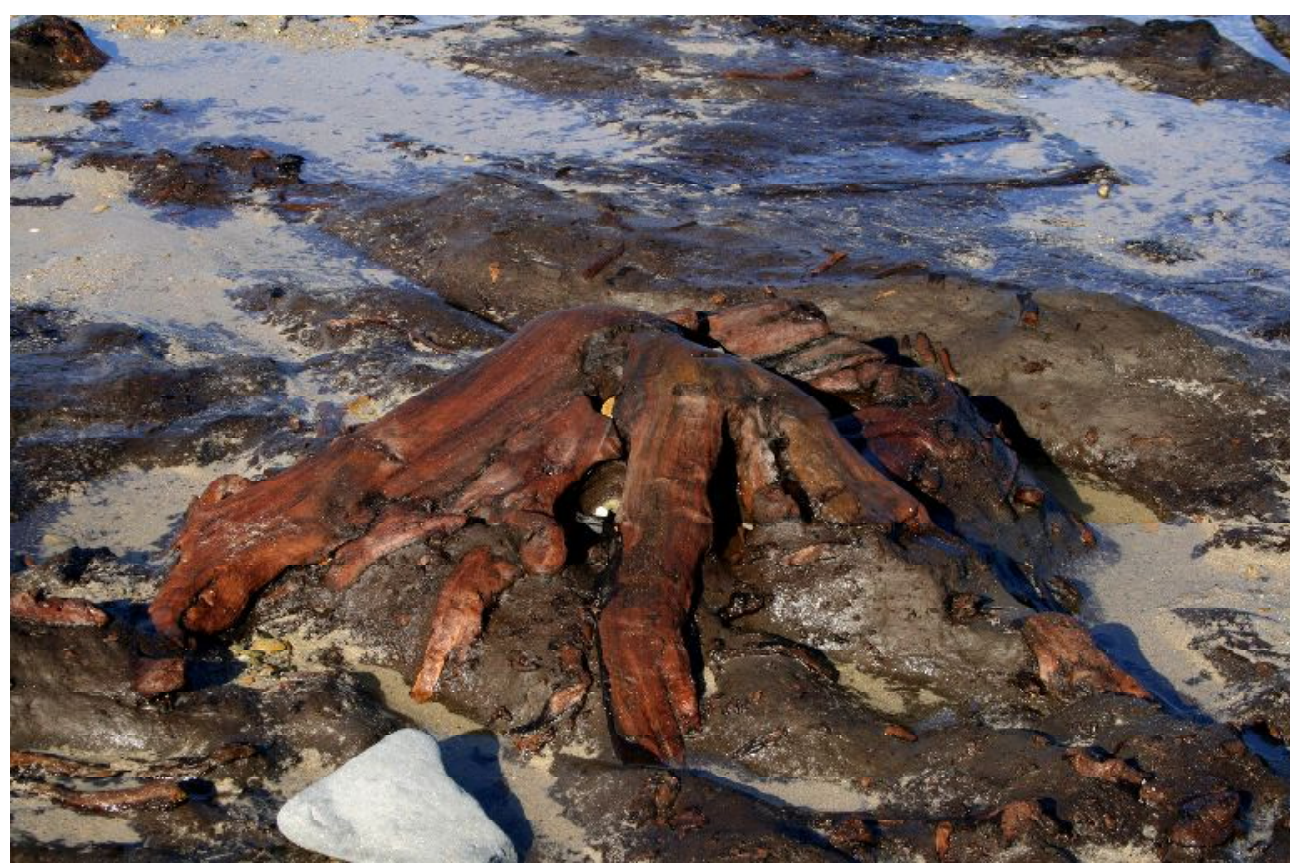

Fig. 3. Bosque de Arealonga 


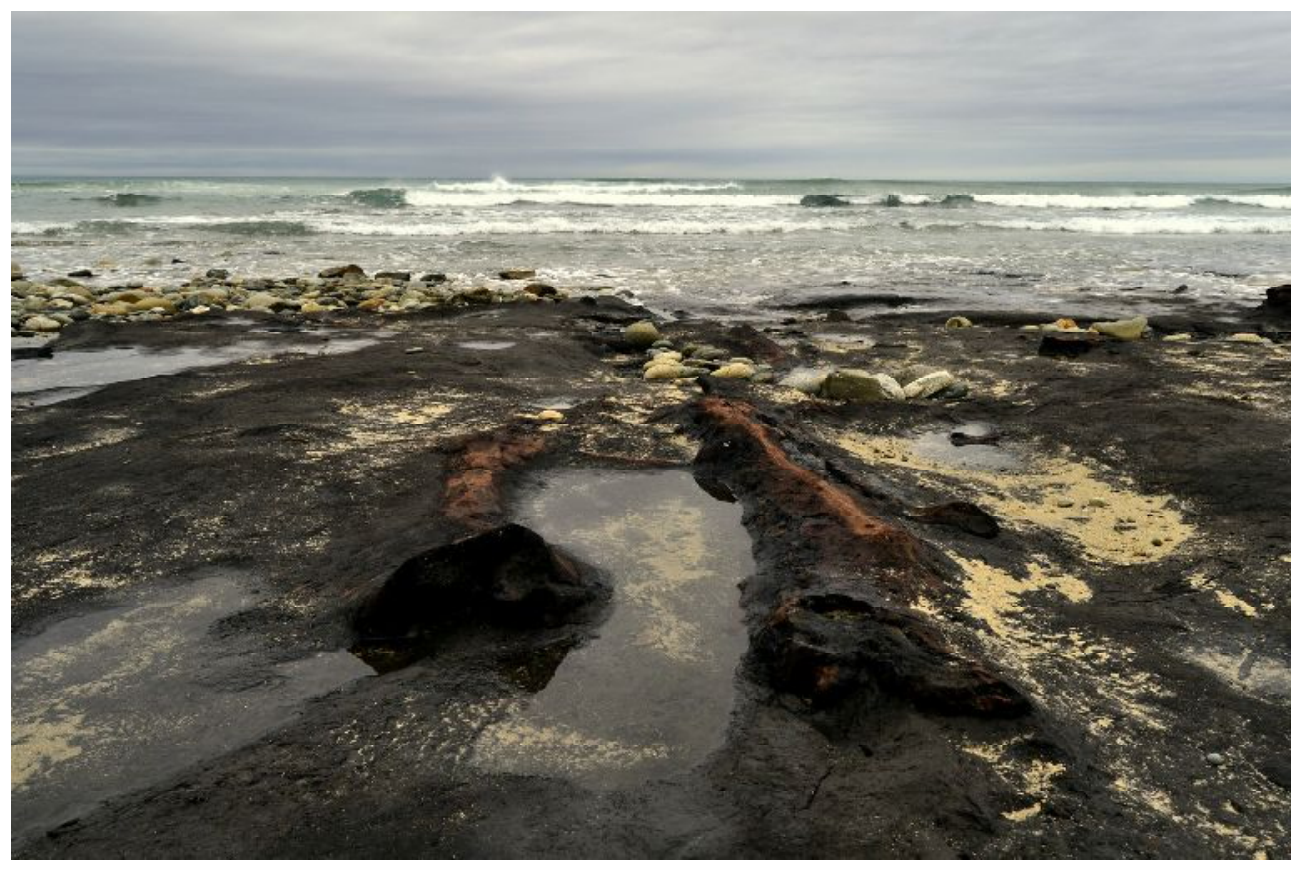

Fig. 4. Bosque de Ponzos

En los últimos 10000 años asistimos a una acelerada subida del nivel del mar con su cohorte de dunas costeras precediéndola. Esta invasión de la costa por trenes de dunas convirtieron la costa en un medio cada vez mas hostil, afectado en las zonas de costa rocosa por las olas y en el resto batido por el viento y las tormentas de arena durante los temporales (Figs. 5a,b,c). Se exceptúa la entrada de las rías donde desembocaban los cursos de agua mas caudalosos y que darían a esta parte de la costa un aspecto muy similar al actual de las Fragas del Eume. 

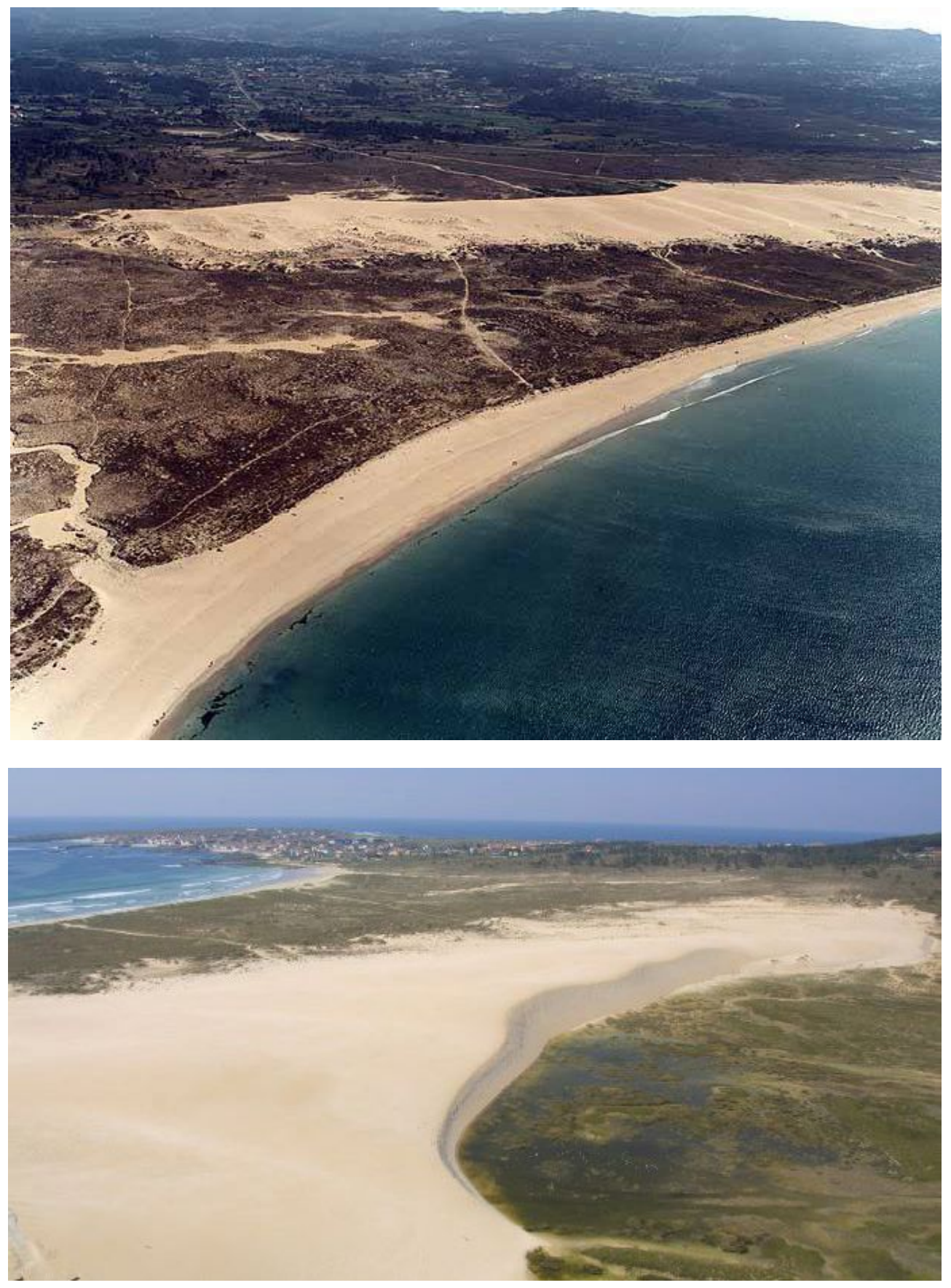


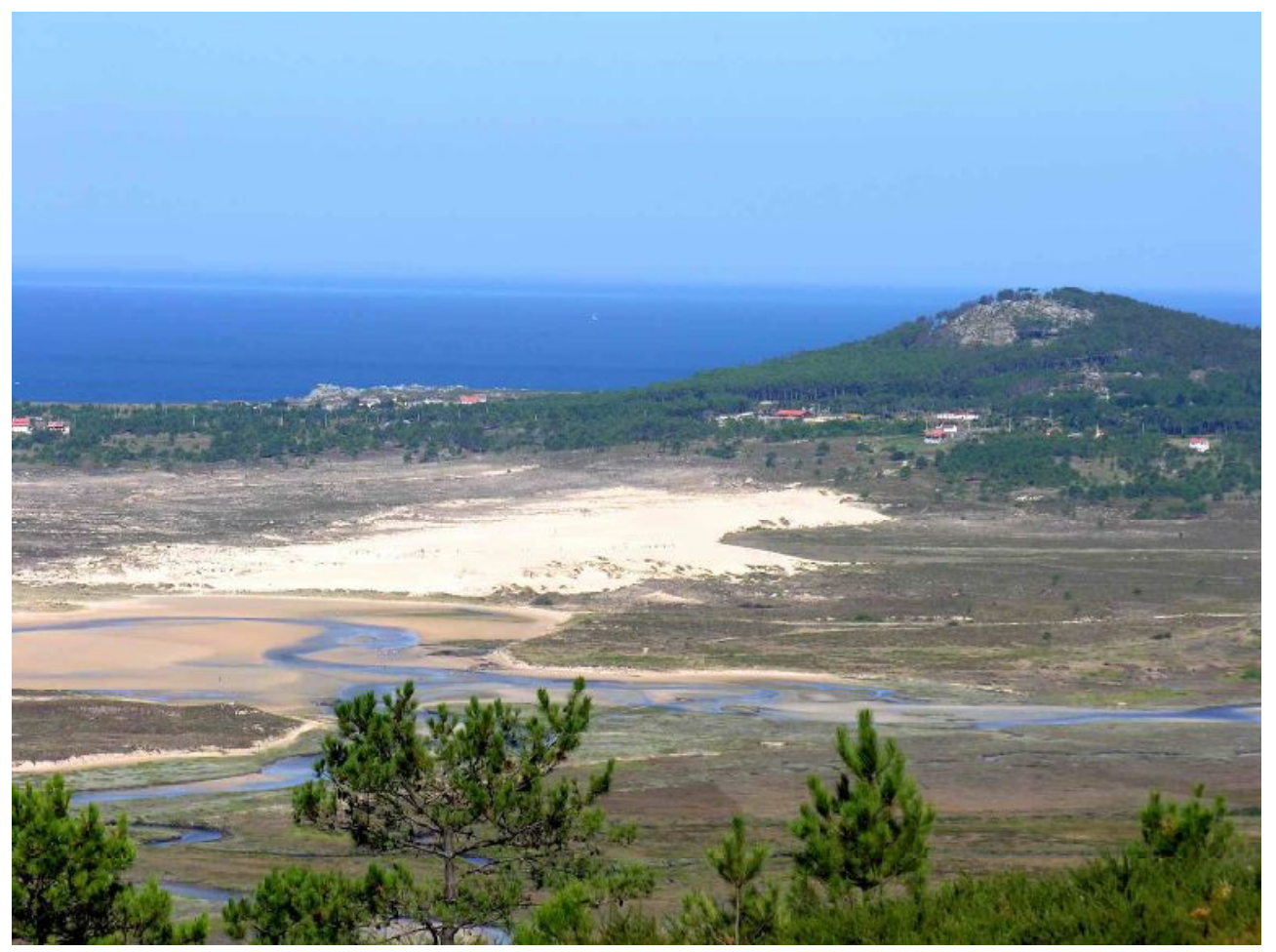

Fig. 5 a,b,c. Las dunas de Corrubedo es un buen ejemplo de como sería la costa gallega hace 9000 años.

Los últimos descubrimientos en especial las espectaculares cuevas descubiertas en zonas graníticas de Galicia (Vaqueiro 2017), han permitido expandir los posibles hábitats cubiertos durante la Prehistoria. Esquemáticamente se pueden distinguir 3 tipos de cuevas no calizas: de fisura, de bloques y cacholas (tafone) (Vidal Romani y Vaqueiro 2007; Vaqueiro 2017). En ellas no se han encontrado restos óseos fósiles debido al factor geoquímico limitante ( $\mathrm{pH}$ ácido) aunque si numerosos restos de cerámica cuya datación en los casos de mayor antigüedad coinciden con los tiempos de Elba (Sanjurjo Sánchez et. al., 2013). Este hecho ha permitido expandir el modelo de ocupación del territorio a prácticamente la totalidad de Galicia y en especial a las zonas graníticas en las que hasta el momento (Fig. 6) existía una gran falta de información. Aparentemente la ocupa- 


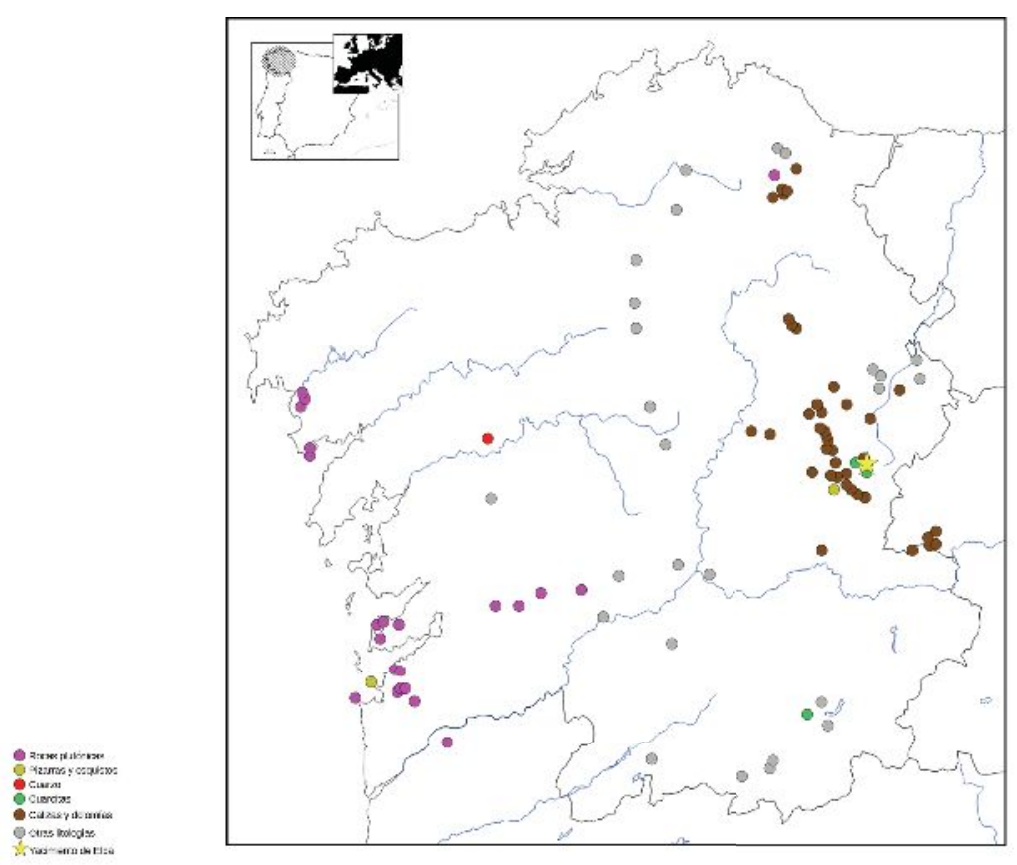

Fig. 6. Localización de cuevas habitadas en Galicia hace 10000 años.

ción humana en la época de Elba era muy diferente en las zonas montañosas costeras que en las interiores. Mientras en Boivão (Portugal), Serra do Galiñeiro, Cunchosa, Adeghas, Louro, y Pindo los sistemas de graníticos de cavidades estaban todos ellos ocupados, hay que suponer que estacionalmente, por los primeros pastores al modo de Elba, en Courel la ocupación humana (Eirós, A Ceza), pareció verse limitada al perímetro de menor altitud de la zona montañosa quedando reservada la zona mas interna y por tanto la que alcanza las cotas topográficas mas elevadas, a las poblaciones de oso pardo que por sus dimensiones pudo ser el animal totémico en la zona. No debería sorprendernos esta distribución pues en cierto modo es lo que continúa ocurriendo en la actualidad.

\section{BIBLIOGRAFÍA}

GONZALEZ FORTES, G., GRANDAL D'ANGLADE, A., VIDAL ROMANÍ, J.R.. Y HOFREITER, M. (2017). Genetic analysis of the individual of chan do lindeiro: characterization of her mitogenome and situation of the sample in the european paleogenic context. Cadernos do Laboratorio Xeolóxico de Laxe. 39, pp. 111-128.

GRANDAL-D'ANGLADE， AURORA Y VIDAL GOROSQUIETA, AMALIA (2017). Isotopic characterization of Elba, the mesolithic woman of Chan do Lindeiro (Pedrafita, Lugo, Iberian Peninsula). Cadernos do Laboratorio Xeolóxico de Laxe. 39, pp. 87-108. 
RAILSBACK, B, LIANG F, VIDAL ROMANÍ J. R, GRANDALD'ANGLADE A, VAQUEIRO RODRÍGUEZ M, SANTOS FIDALGO L, FERNÁNDEZ MOSQUERA D, CHENG H, LAWRENCE EDWARDS R. (2011). Petrographic and isotopic evidence for Holocene long-term climate change and shorter-term environmental shifts from a stalagmite from the Serra do Courel of northwestern Spain, and implications for climatic history across Europe and the Mediterranean. Palaeogeography, Palaeoclimatology, Palaeoecology, t. 305, 172-184.

SANIN MATÍAS, M. Y SERRULLA F. (2017). Informe antropológico forense: aproximación facial. Cadernos do Laboratorio Xeolóxico de Laxe. 39, pp. 71-86.

SANJURJO SÁNCHEZ J.; VIDALROMANÍ, J. R.; VAQUEIRO RODRÍGUEZ, M.; COSTAS VAZQUEZ R.; GRANDAL D'ANGLADE, A. (2013). TL estimation of ages of pottery fragments recovered from granite caves in the NW coast of Spain. Cadernos do Laboratorio Xeolóxico de Laxe, 37,7388.

SANTOS L.; VIDAL ROMANÍ J. R. \& JALUT G. (2000). History of vegetation during the Holocene in the Courel and Queixa Sierras, Galicia, northwest Iberian Peninsula. Journal of Quaternary Science, 15 (6) 621-632.

STEELMAN, K.L.; DE LOMBERA-HERMIDA, A.; VIÑAS-VILLAVERDÚ R.; RODRÍGUEZ-ÁLVAREZ X.P.; CARRERA-RAMÍREZ F.; RUBIO-MORA,
A. AND FÁBREGAS-VALCÁRCEL R. (2017). Cova Eirós: an integrated approach to dating the earliest known cave art in NW Iberia. Radiocarbon, vol. 59. Nr 1, pp. 151-164. DOI:10.1017/ RDC.2017.4

SERRULLA, F., SANIN MATIAS, M. (2017). Forensic antropological report of Elba. Cadernos do Laboratorio Xeolóxico de Laxe. 39, pp. 33-70.

VAQUEIRO-RODRÍGUEZ, M. COSTASVÁZQUEZ R., COSTAS-SUÁREZ D., VIDAL-ROMANI, J.R. (2017). Características morfológicas de la Sima del Uro (NO, España). Morphologic map of "Sima del Uro" (NW, Spain). Cadernos do Laboratorio Xeolóxico de Laxe. 39, pp. 23-34.

VAQUEIRO M. (2017). Cavidades naturales en rocas magmáticas. Las cuevas en rocas plutónicas. Tesis doctoral. Universidad de Coruña. 492 pp. (inédita)

VIDAL GOROSQUIETA, AMALIA; GRANDAL-D'ANGLADE, AURORA; VAQUEIRO-RODRÍGUEZ $M$ and VIDAL-ROMANI, J.R. (2016). Galician aurochs: a morphologic, metric and isotopic study. 1st IMERP.XIV EJIP. New perspectives on the Evolution of Phanerozoic Biotas and ecosystems. pp.167.

VIDAL ROMANI J.R. (2015). La Geología de Galicia o como armar un rompecabezas. Discurso de Ingreso en la Real Academia Gallega de Ciencias. Santiago de Compostela.

VIDAL ROMANÍ J. R.; VAQUEIRO M. (2007). Types of granite cavities and associated speleothems: genesis and evolution. Nature Conservation 63, 41-46. 